\title{
OPEN CAPACITATED ARC ROUTING PROBLEM BY HYBRIDIZED ANT COLONY ALGORITHM
}

\author{
Bilal Kanso ${ }^{1, *}$, Ali Kansou ${ }^{1}$ and Adnan Yassine ${ }^{2}$
}

\begin{abstract}
The Open Capacitated Arc Routing Problem OCARP is a well-known NP-hard real-world combinatorial optimization problem. It consists of determining optimal routes for vehicles in a given service area at a minimal cost distance. The main real application for OCARP is the Meter Reader Routing Problem (MRRP). In MRRP problem, each worker in the electric (or gas) company must visit and read the electric (or gas) meters to a set of customers by starting his route from the first customer on his visit list and finishing with the last one. The worker leaves where he wants once all the associated customers have been visited. In this paper, a metaheuristic called an Hybridized Ant Colony Algorithm (HACA) is developed and hybridized with a local search algorithm that involves the 2-opt, Swap, Relocate and Cross-exchange moves to solve OCARP problem. Computational results conducted on five different sets of OCARP-instances showed that our proposed algorithm HACA has reached good and competitive results on benchmark instances for the problem.
\end{abstract}

Mathematics Subject Classification. 90C32, 90C26, 90C59.

Received June 16, 2019. Accepted March 2, 2021.

\section{INTRODUCTION}

The Open Capacitated Arc Routing Problem (OCARP) is a special variant of the Capacitated Arc Routing Problem (CARP) where a vehicle starts at a given edge and does not return to the depot after servicing the last edge on a route. In the last ten years, OCARP received attention in the operation research literature. Indeed, it turns out that it is suitable to model for a wide range of real-life applications such as package delivery problem, newspaper home delivery problem, school bus problem or meter reader routing problem [14, 21]. In such applications, the employees use their own vehicles to serve edges and are not required to return to depot after completing service of edges. The objective is to design a set of routes of total minimum distance travelled by all vehicles. The authors in [17] showed that OCARP is NP-hard and proposed a formal definition of this variant of CARP which can be described as follows: (1) the graph $G\left(N, E, E_{r}\right)$ is directed where $N$ is the set of nodes, $E$ is the set of all edges and $E_{r}$ is the set of required edges (called tasks) with demands strictly positive; (2) a fleet of $M$ identical vehicles of capacity $Q$ where $M$ is predefined; (3) each task must be served by one direction and by one and only one vehicle; (4) every route (made by a vehicle) starts from the first task, serves

Keywords. Open Capacitated Arc Routing Problem, metaheuristic, Ant Colony Algorithm, local search, Simulated Annealing.

1 Department of Computer Science, Lebanese University, Beirut, Lebanon.

2 Normandie University, 25 rue Philippe Lebon, Le Havre, France.

*Corresponding author: bilal.kanso@hotmail.com, bilal_kanso@hotmail.com 
an order of tasks and finally ends at the last served task; (5) routes must verify the capacity constraint. Thus, OCARP aims to find at most $M$ set of feasible routes with minimum total cost distance.

The authors in [17] proposed a heuristic based on a Reactive Path-Scanning (RPS) with ellipse rule to solve OCARP and found the first known solutions. In [18], the authors developed an exact branch-and-bound algorithm and improved the best known lower bounds. In [19], the authors developed a greedy randomized adaptive search procedure (GRASP) with evolutionary path-relinking which slightly improved the solutions found in [18]. In the last work on OCARP [1], the authors developed Hybrid Genetic Algorithm (HGA) and provided the best metaheuristic solutions for OCARP.

In this paper, we introduce a new approach based on Hybrid Ant Colony Algorithm (HACA). The representation of ants is considered as a giant route containing all tasks of $E_{r}$ in such a way each task is served in one direction. Each ant is represented by only one vehicle and does not verify the capacity constraint. An evaluation procedure adapted from the splitting algorithm introduced in [16] is developed to make the ants feasible and then evaluate them. Our method is hybridized with a local search procedure based on Simulated Annealing (SA) which operates by sequentially performing moves to existing feasible solutions in order to eventually improve them as the search progresses [9]. The main move used in SA algorithm is the cross-exchange which is adapted for OCARP for the first time in this work. Finally, experiments that we have conducted are capable of solving significantly larger instances of the OCARP existing in the literature $[2,6]$ and found good (some are optimal) solutions without expending much computational execution time.

This paper is organized as follows. Section 2 describes our studied problem OCARP. Section 3 presents our hybrid HACA algorithm which is a combination of an ant colony method with a local search procedure based on Simulated Annealing (SA) to efficiently solve OCARP. Section 4 shows the computational experiments that we conducted and analyses the results. Finally, a conclusion about this work is summarized in Section 5.

\section{Problem DESCRIPTION}

The Open Capacited Arc Routing Problem (OCARP) was introduced and described in [17]. In this variant of CARP, each vehicle starts in its first task (required edge with a demand strictly positive) and finish at the last visited task. The number of vehicles is limited and each task can be visited once by a specific vehicle. The capacity of vehicles $Q$ is predefined and homogeneous for all used vehicles. A travelling distance $C_{p q}$ is considered between each couple of tasks $(p, q)$. A feasible solution is a set of feasible routes where each feasible route is a sequence of tasks visited and serviced by the same vehicle considering the capacity constraint. The main objective of OCARP is to minimize the total travelling distance by all feasible routes in the feasible solution. Other objective functions and constraints can be considered, like time windows and heterogeneous vehicles, but they are out of our work.

Therefore, the details of OCARP can be described as follows: let $K$ be a set of $M$ identical vehicles and $G=\left(N, E, E_{r}\right)$ be a graph where $N$ is the node set that represents the starting and ending points of edges and $E=E_{r} \cup E_{n r}$ is the edge set. $E_{r}$ contains the required edges (called tasks) where each task $p$ represents an edge with demand $d_{p}>0$ and must be served by one and only one vehicle $k \in K$. $E_{n r}$ is the set of all no required edges with demand zero. Firstly, a travelling distance $D_{x y}$ between nodes is associated to each couple $(x, y) \in N \times N$. The cost $C_{p q}$ between two tasks $p=(x, y)$ and $q=(z, t)$ will be calculated by Dijkstra's algorithm from the final node $y$ of $p$ to the origin node $z$ of $q$. The notation of the cost $C_{p}=D_{x y}$ is used to traverse the task $p=(x, y), \forall p \in E_{r}$ where $(x, y) \in N \times N$. The notation $\operatorname{Cost}(S)$ is used to get the total cost of a given solution $S$ which is considered as the total sum of costs of all routes involved in the solution $S$.

\section{AN ANT COLONY ALGORITHM FOR THE OCARP}

We propose an ant colony algorithm to solve the OCARP. Ant Colony Optimization algorithms (ACO) are global search optimization techniques based on the foraging behaviour of real ants which have been found to be very effective for solving the travelling salesman problem (TSP) $[3,5,15]$ and the capacitated arc routing problem (CARP) [7]. In this work, we construct an initial solution $S_{0}$ by the basic Path Scanning algorithm 
(PS) [17]. The ACO algorithm uses a colony of $N_{a}$ artificial ants where each ant $g$ represents a non-capacitated giant route (order of all different tasks in $E_{r}$ ), i.e. the constraint capacity is not verified. At each iteration of the ACO, every ant starts its route at a random task. Moving of ants depends on two amounts: (1) visibility amount $\mu_{p q}$ which is a constant and represents the inverse of the cost between two tasks $p$ and $q$; (2) pheromone amount $\tau_{p q}$ which is a variable and initially represents the inverse of the cost of initial solution, i.e. $\tau_{p q}=$ $\frac{1}{\operatorname{Cost}\left(S_{0}\right)} \forall p, q \in E_{r}$. The amount of pheromone will be updated at the end of each iteration $t$ by the following rule $\tau_{p q}(t+1)=\rho \tau_{p q}(t)+(1-\rho) \Delta \tau_{p q}$ where $\rho$ is the evaporation parameter in $[0,1]$ and $\Delta \tau_{p q}$ equals to $\frac{1}{\text { Cost(best) }}$ if task $q$ is used just after task $p$ by the ant that gives the best solution best and zero otherwise.

The following subsection explains how to find an initial solution $S_{0}$ by Path Scanning heuristic. The second subsection presents the evaluation procedure to split and make feasible a giant route. The four moves used in the local search procedure and the detailed algorithm are explained in the third subsection. Finally, the components and the pseudo-code of the ACO algorithm are presented in the last subsection.

\subsection{Path Scanning heuristic for OCARP problem}

The classic Path Scanning heuristic (PS) is used for building the feasible initial solution $S_{0}$ of our proposed ACO metaheuristic. PS constructs the routes in a sequential manner, i.e. it begins to build the first route without exceeding the capacity of the vehicle used on this route. Once a route is constructed, PS tries to build other routes in the same way. Note that, when the number of vehicles exceeds the predefined number of vehicles $M$, then the obtained solution is not taken into consideration.

PS builds each feasible route as follows. Firstly, PS selects a random task to be the starting one of the constructed route. Then, it chooses the next task $s$ to add after the last selected task $l$ among the candidate tasks using the nearest neighbour rule. If there are more than one task that meet the capacity constraint and have the same cost from $l$, then one rule of the following five rules is applied: (1) minimize $\frac{C_{s}}{d_{s}} ;(2)$ maximize $\frac{C_{s}}{d_{s}} ;(3)$ minimize the cost back to a virtual depot 0 ; (4) maximize the cost back to a virtual depot 0 ; and (5) use rule (3) if the vehicle has used more than half of its capacity $Q$, and rule (4) otherwise.

The used virtual depot 0 represents the node 1 in the node set $N$ in each graph instance $G$ used in the numerical results (instance files are explained in Sect. 4). Indeed, the node 1 is used to be the first depot in the basic instance files of CARP with single depot. Furthermore, in our experiments each node in $N$ has been tested to be the virtual depot 0 , but no better results are attained. The heuristic PS is repeated 1000 times for each instance and the best costs of feasible obtained solutions are recorded (see results in Sect. 4). Finally, the best solution with the minimal total cost distance is the initial feasible solution $S_{0}$ used for ACO.

\subsection{Evaluation procedure}

The split procedure is commonly used for capacitated arc routing problem CARP [10,16]. We adopt it to make each ant feasible by splitting it into a set of feasible routes (i.e. a feasible OCARP solution) and to evaluate its fitness. Given an ant $g=\left(g_{1}, g_{2}, \ldots, g_{r}\right)$ of $r$ tasks, we construct an acyclic auxiliary graph $H(T, Z)$ with $r+1$ nodes, where $T$ is a vertex set containing nodes indexed from 0 to $r$, and $Z$ is a set of evaluated directed arcs. 0 is a fictitious node and every node $i \in T \backslash\{0\}$ represents the task $g_{i}$ in $g$. An arc $(i, j)$ such that $i<j$, is associated to $Z$ if it represents a feasible route $i$.e. a subsequence of tasks $\left(g_{i+1}, \ldots, g_{j}\right)$ where $\sum_{k=i+1}^{j} d_{g_{k}} \leq Q$. Every $\operatorname{arc}(i, j) \in Z$ will be evaluated by the $\operatorname{cost} z_{i j}$ which is calculated by the following rule:

$$
z_{i j}=\left\{\begin{array}{lll}
C_{g_{j}}+\sum_{k=i}^{j-2}\left[C_{g_{k+1}}+C\left(g_{k+1}, g_{k+2}\right)\right] & \text { if } & j-i \geq 2 \\
C_{g_{j}} & \text { if } & j-i=1 .
\end{array}\right.
$$


For each ant $g$, the shortest path $\mathrm{SP}^{g}$ from node 0 to node $r$ of $T$ in graph $H$ by using at most $M$ arcs of $Z$, is calculated by Algorithm 1. This algorithm is an adaptation of Bellman-Ford algorithm [4] to take into consideration at most $M$ arcs on the target shortest path. Hence, the shortest path $\mathrm{SP}^{g}$ represents the optimal partition of the corresponding ant $g$ (giant route). The fitness cost of the optimal solution is denoted by $\operatorname{Cost}\left(S^{g}\right)$ where $S^{g}$ is the corresponding feasible OCARP solution from ant $g$ and $\operatorname{Cost}\left(S^{g}\right)=\sum_{(i, j) \in \mathrm{SP}^{g}} z_{i j}$.

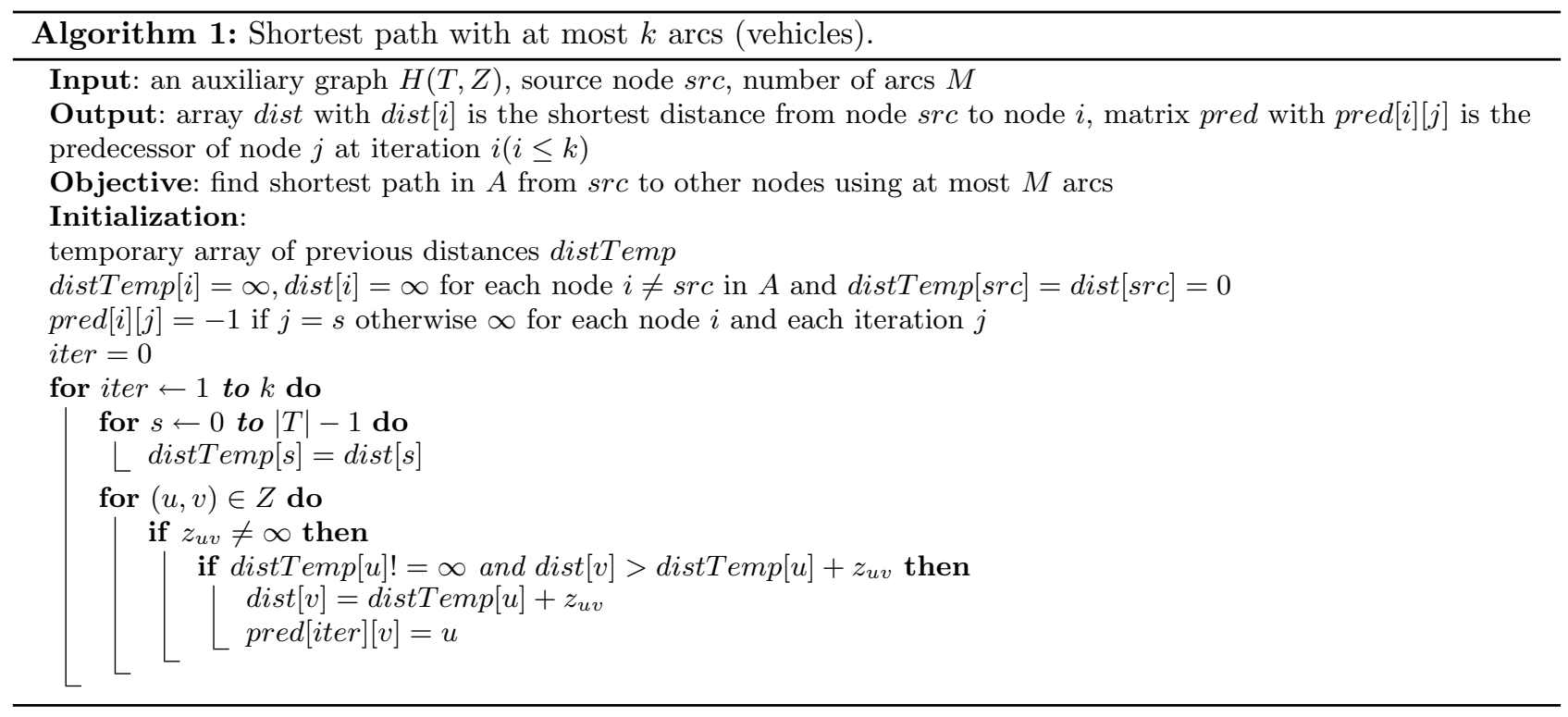

Table 1 illustrates an example of six tasks $g_{1}, g_{2}, g_{3}, g_{4}, g_{5}$ and $g_{6}$ required to explain the details of our split procedure. The number of vehicles is $M=2$ and the vehicle capacity is $Q=20$. Suppose that the costs among tasks are given as follows: $C_{g_{1} g_{2}}=33, C_{g_{2} g_{3}}=38, C_{g_{3} g_{4}}=27, C_{g_{4} g_{5}}=32$ and $C_{g_{5} g_{6}}=30$.

Figure 1A illustrates the auxiliary graph $H(T, Z)$ associated to the following giant route $g$ (or ant $g$ ):

\begin{tabular}{|l|l|l|l|l|l|}
\hline$g_{1}$ & $g_{2}$ & $g_{3}$ & $g_{4}$ & $g_{5}$ & $g_{6}$ \\
\hline
\end{tabular}

As two examples, the arc $(0,1)$ represents a feasible route containing $g_{1}$ (i.e. only $g_{1}$ is served) with cost $z_{01}=C_{g_{1}}=22$ and the arc $(0,2)$ means that a vehicle serves $g_{1}$ then $g_{2}$ with cost $z_{02}=C_{g_{1}}+C_{g_{1} g_{2}}+C_{g_{2}}=$ $22+33+20=75$. Figures $1 \mathrm{~B}$ and $1 \mathrm{C}$ illustrate two routes generated from the auxiliary graph using our adapted Bellman-Ford algorithm presented in Algorithm 1. For the proposed example, $\mathrm{SP}^{g}$ is composed of two arcs $(0,2)$ and $(2,6)$ and then the corresponding feasible solution $S^{g}$ is composed of two routes presented respectively in Figures 1B and 1C. The cost of $S^{g}$ is $\operatorname{Cost}\left(S^{g}\right)=z_{02}+z_{26}=75+175=250$.

TABLE 1. Information about an example with 6 tasks.

\begin{tabular}{lllllll}
\hline \hline Task & $g_{1}$ & $g_{2}$ & $g_{3}$ & $g_{4}$ & $g_{5}$ & $g_{6}$ \\
\hline Demand & 8 & 8 & 5 & 6 & 4 & 5 \\
Cost & 22 & 20 & 20 & 25 & 26 & 15 \\
\hline
\end{tabular}




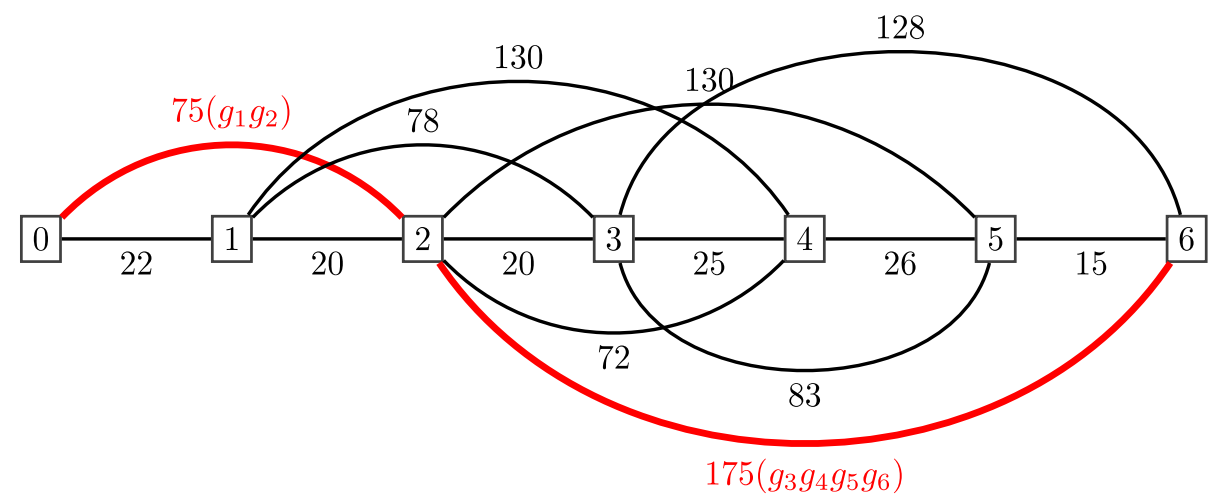

(A)

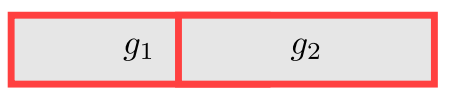

(B)

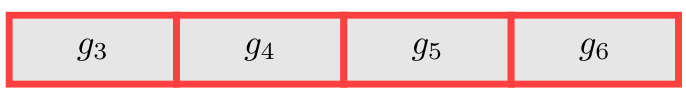

(C)

Figure 1. Example of the split procedure. (A) Auxiliary graph. (B) First route $(\operatorname{cost}=75)$. (C) Second route $(\operatorname{cost}=175)$.

\subsection{Local search procedure}

In order to improve the obtained feasible solutions by the proposed ACO algorithm, we apply the Simulated Annealing algorithm (SA) in the local search procedure. The SA is a metaheuristic which is commonly used to prevent the optimization algorithm to quickly fall into local minimum $[12,20]$. It is based on the random acceptance strategy with certain probability. The local search moves that we implemented in the SA algorithm are the following:

(a) Swap move: two versions of this move have been implemented. The first one consists of exchanging two distinct tasks $p$ and $q$ belonging to the same feasible route. The second one consists of exchanging two distinct tasks belonging to two different feasible routes. For each call of the "Swap" move, we choose randomly the way to apply it.

(b) Relocate move: this move consists of removing one task from a position $i$ on a route $R$ and putting it in another position $j$ chosen in the same route $R$ or in another route $R^{\prime}$.

(c) 2-opt move: this move consists of choosing two positions $i$ and $j$ in a given route $R$ and then re-organizes $R$ by replacing the sequence of tasks $(i, i+1, \ldots, j)$ by the sequence $(i n v(j), i n v(j-1), \ldots, i n v(i))$ where $i n v(t)$ represents the inverse task for the task $t$. For each call of the "2-opt" move, we apply it to every route into the considered solution.

(d) Cross-exchange move: this move involves two routes $R_{1}$ and $R_{2}$ where it chooses a task in a position $i$ of $R_{1}$ and a task in a position $j$ of $R_{2}$. It generates two new routes $R_{1}^{\prime}$ and $R_{2}^{\prime}$ as follows: $R_{1}^{\prime}$ takes the first part of $R_{1}$ from first task to task located at position $i$ completed by another part of the route $R_{2}$ starting with the task located at position $j . R_{2}^{\prime}$ is built in the same way and that by linking the remaining first part of $R_{2}$ with the second remaining part of $R_{1}$.

Note that all moves are applied only if the resulting solutions are feasible. The Simulated Annealing method in the local search procedure is presented in Algorithm 2. 


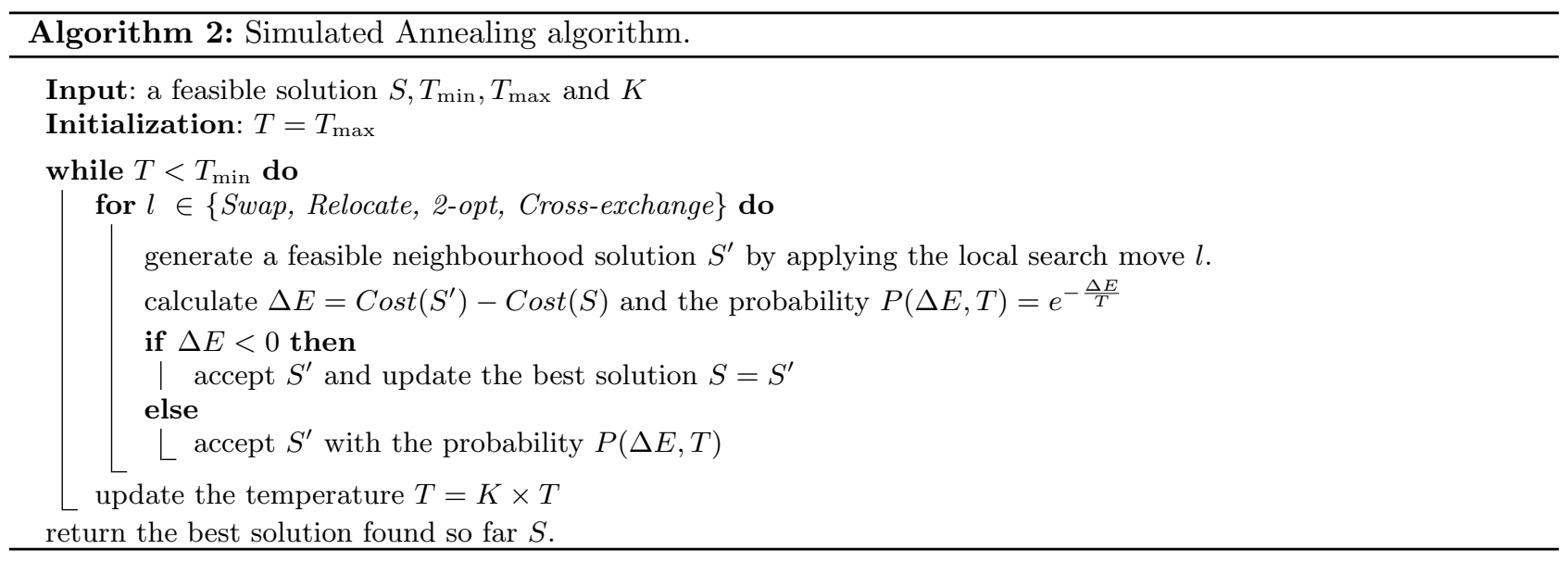

\subsection{The proposed HACA algorithm}

Suppose that Visited $_{g}$ is the set of already visited tasks by ant $g$. An ant $g$ moves from the last visited task $i$ to another task $j$ by choosing the closest task $j$ ( $j \notin V i$ sited $d_{g}$ ) to $i$ if the random variable $q$ is strictly less than $q_{0}$. Otherwise, it is chosen by using the following probability rule:

$$
P_{i j}^{g}= \begin{cases}\frac{\tau_{i j}^{\alpha} \times \mu_{i j}^{\beta}}{\sum_{k \in \text { isitedg }_{g}} \tau_{i k}^{\alpha} \times \mu_{i k}^{\beta}} & \text { if } j \notin \text { Visited }_{g} \\ 0 & \text { otherwise }\end{cases}
$$

where $\alpha$ and $\beta$ are respectively the pheromone and visibility parameters, $q_{0}$ is a constant parameter belongs to $[0,1]$ and $q$ is a random parameter in $[0,1]$. The proposed HACA algorithm is shown in Algorithm 3.

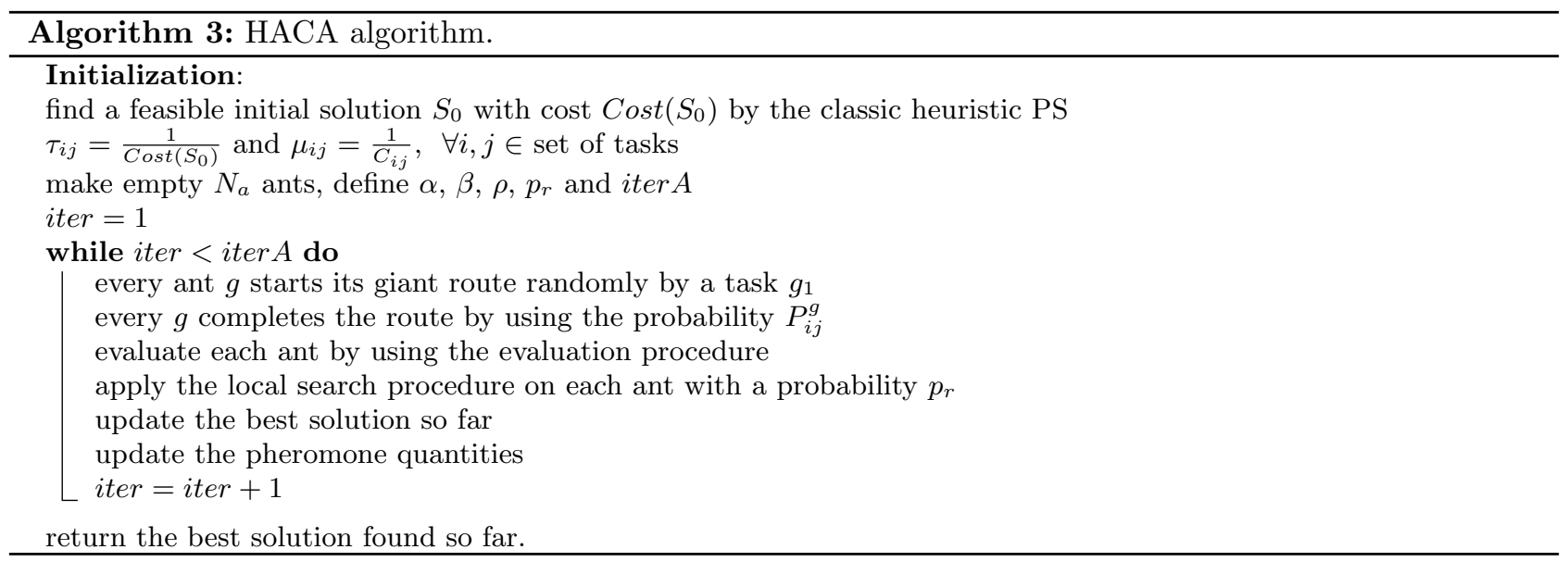


TABLE 2. Results of the HACA algorithm on ogdb's instances.

\begin{tabular}{lllllllllll}
\hline \hline Instance & $M$ & lb & RPS & GRASP & HGA & HACA & G-R & G-G & G-H & $T$ \\
\hline ogdb1 & 7 & 252 & 252 & 252 & 252 & 252 & 0 & 0 & 0 & $<1$ \\
ogdb2 & 8 & 291 & 291 & 291 & 291 & 291 & 0 & 0 & 0 & $<1$ \\
ogdb3 & 7 & 233 & 233 & 233 & 233 & 233 & 0 & 0 & 0 & $<1$ \\
ogdb4 & 6 & 238 & 238 & 238 & 238 & 238 & 0 & 0 & 0 & $<1$ \\
ogdb5 & 8 & 316 & 316 & 316 & 316 & 316 & 0 & 0 & 0 & $<1$ \\
ogdb6 & 7 & 260 & 260 & 260 & 260 & 260 & 0 & 0 & 0 & $<1$ \\
ogdb7 & 7 & 262 & 262 & 262 & 262 & 262 & 0 & 0 & 0 & $<1$ \\
ogdb8 & 12 & 210 & 210 & 210 & 210 & 210 & 0 & 0 & 0 & $<1$ \\
ogdb9 & 12 & 219 & 219 & 219 & 219 & 219 & 0 & 0 & 0 & $<1$ \\
ogdb10 & 6 & 252 & 252 & 252 & 252 & 252 & 0 & 0 & 0 & $<1$ \\
ogdb11 & 7 & 356 & 360 & 358 & 358 & 358 & 0 & 0 & 0 & $<1$ \\
ogdb12 & 9 & 336 & 336 & 336 & 336 & 336 & 0 & 0 & 0 & $<1$ \\
ogdb13 & 8 & 509 & 509 & 509 & 509 & 509 & 0 & 0 & 0 & $<1$ \\
ogdb14 & 7 & 96 & 96 & 96 & 96 & 96 & 0 & 0 & 0 & $<1$ \\
ogdb15 & 6 & 56 & 56 & 56 & 56 & 56 & 0 & 0 & 0 & $<1$ \\
ogdb16 & 7 & 119 & 119 & 119 & 119 & 119 & 0 & 0 & 0 & $<1$ \\
ogdb17 & 7 & 84 & 84 & 84 & 84 & 84 & 0 & 0 & 0 & $<1$ \\
ogdb18 & 7 & 158 & 158 & 158 & 158 & 158 & 0 & 0 & 0 & $<1$ \\
ogdb19 & 5 & 45 & 45 & 45 & 45 & 45 & 0 & 0 & 0 & $<1$ \\
ogdb20 & 6 & 105 & 105 & 105 & 105 & 105 & 0 & 0 & 0 & $<1$ \\
ogdb21 & 8 & 149 & 149 & 149 & 149 & 149 & 0 & 0 & 0 & $<1$ \\
ogdb22 & 10 & 191 & 191 & 191 & 191 & 191 & 0 & 0 & 0 & $<1$ \\
ogdb23 & 12 & 223 & 223 & 223 & 223 & 223 & 0 & 0 & 0 & $<1$ \\
\hline
\end{tabular}

\section{EXPERIMENTS AND RESULTS}

\subsection{Benchmark instances and experiments}

For our computational experiments, we use five sets of standard test instances: ogdb, oAi, oBi, oval and oegl. These sets are extensions of standard sets of $\mathrm{CARP}^{1}$ instances. Thus, our OCARP Benchmark includes 23 ogdb [8], $32 \mathrm{oAi}, 24 \mathrm{oBi}, 34$ oval [2] and $24 \mathrm{oegl}$ [6], totalling 137 instances. $M=M^{*}+2$ is the number of vehicles used in our experiments where $M^{*}$ represents the minimal value of the number of vehicles required for finding a feasible solution reported in [17]. The experiments were implemented in Java and run on a $2.6 \mathrm{GHz}$ Dual Core computer with a memory of 16 GB under Windows Ten.

Throughout the section, the results of our experiments are compared with respect to three methods from literature: RPS (Reactive Path-Scanning with ellipse rule) heuristic [17], GRASP (Greedy Randomized Adaptive Search Procedure with path relinking) metaheuristic [19] and HGA (Hybrid Genetic Algorithm) [1]. Tables 2-6 show the obtained results of all instances after running our algorithm. For each table, the columns correspond respectively to: (1) problem name instance; (2) the number of vehicles $M$; (3) lower bound (lb) which is the corresponding bound to the best feasible solution as reported in [17]; (4) cost obtained by RPS method; (5) cost obtained by GRASP method; (6) cost obtained by HGA method; (7) cost obtained by our hybrid ant algorithm (HACA); (8) gap in percentage $(\mathrm{G}-\mathrm{R}(\%))$ of RPS cost compared to HACA; (9) gap in percentage $(\mathrm{G}-\mathrm{G}(\%))$ of GRASP cost compared to HACA; (10) gap in percentage $(\mathrm{G}-\mathrm{H}(\%))$ of HGA cost compared to HACA; (11) running time in seconds for HACA $(\mathrm{T})$. The gaps $\mathrm{G}-\mathrm{R}(\%), \mathrm{G}-\mathrm{G}(\%)$ and $\mathrm{G}-\mathrm{H}(\%)$ are computed by the following rule: $\mathrm{G}-\mathrm{X}=\frac{\mathrm{X}-\mathrm{HACA}}{\mathrm{X}} \times 100$ with $\mathrm{X} \in\{\mathrm{RPS}$, GRASP, $\mathrm{HGA}\}$.

\footnotetext{
${ }^{1}$ CARP's instances can be found at http://www.uv.es/Belengue/carp.html; http://www.hha.dk/sanw.
} 
TABLE 3. Results of the HACA algorithm on oAi's instances.

\begin{tabular}{lllllllllll}
\hline \hline Instance & $M$ & lb & RPS & GRASP & HGA & HACA & G-R & G-G & G-H & $T$ \\
\hline oAi10A & 6 & 43 & 43 & 43 & 43 & 43 & 0 & 0 & 0 & $<1$ \\
oAi10B & 5 & 43 & 43 & 43 & 43 & 43 & 0 & 0 & 0 & $<1$ \\
oAi10C & 4 & 43 & 43 & 43 & 43 & 43 & 0 & 0 & 0 & $<1$ \\
oAi10D & 3 & 43 & 45 & 43 & 43 & 43 & 4.44 & 0 & 0 & $<1$ \\
oAi13A & 10 & 85 & 85 & 85 & 85 & 85 & 0 & 0 & 0 & $<1$ \\
oAi13B & 6 & 85 & 85 & 85 & 85 & 85 & 0 & 0 & 0 & $<1$ \\
oAi13C & 4 & 85 & 88 & 85 & 85 & 85 & 3.41 & 0 & 0 & $<1$ \\
oAi13D & 4 & 85 & 91 & 85 & 85 & 85 & 6.59 & 0 & 0 & $<1$ \\
oAi15A & 10 & 92 & 92 & 92 & 92 & 92 & 0 & 0 & 0 & $<1$ \\
oAi15B & 7 & 92 & 92 & 92 & 92 & 92 & 0 & 0 & 0 & $<1$ \\
oAi15C & 5 & 92 & 92 & 92 & 92 & 92 & 0 & 0 & 0 & $<1$ \\
oAi15D & 4 & 92 & 94 & 92 & 92 & 92 & 2.13 & 0 & 0 & $<1$ \\
oAi20A & 13 & 113 & 113 & 113 & 113 & 113 & 0 & 0 & 0 & $<1$ \\
oAi20B & 9 & 113 & 113 & 113 & 113 & 113 & 0 & 0 & 0 & $<1$ \\
oAi20C & 6 & 113 & 113 & 113 & 113 & 113 & 0 & 0 & 0 & $<1$ \\
oAi20D & 5 & 113 & 116 & 113 & 113 & 113 & 2.59 & 0 & 0 & $<1$ \\
oAi24A & 14 & 139 & 139 & 139 & 139 & 139 & 0 & 0 & 0 & $<1$ \\
oAi24B & 9 & 139 & 139 & 139 & 139 & 139 & 0 & 0 & 0 & $<1$ \\
oAi24C & 6 & 139 & 151 & 145 & 145 & 145 & 3.98 & 0 & 0 & $<1$ \\
oAi24D & 5 & 139 & 154 & 148 & 148 & 148 & 3.9 & 0 & 0 & $<1$ \\
oAi27A & 12 & 188 & 188 & 188 & 188 & 188 & 0 & 0 & 0 & $<1$ \\
oAi27B & 8 & 188 & 191 & 188 & 188 & 188 & 1.57 & 0 & 0 & $<1$ \\
oAi27C & 5 & 194 & 202 & 197 & 197 & 197 & 2.48 & 0 & 0 & 1 \\
oAi27D & 4 & 197 & 208 & 202 & 202 & 202 & 2.88 & 0 & 0 & $<1$ \\
oAi31A & 21 & 271 & 274 & 271 & 271 & 271 & 1.1 & 0 & 0 & 7.6 \\
oAi31B & 13 & 271 & 271 & 271 & 271 & 271 & 0 & 0 & 0 & 4.5 \\
oAi31C & 8 & 271 & 282 & 277 & 277 & 277 & 1.77 & 0 & 0 & 5.4 \\
oAi31D & 6 & 271 & 292 & 284 & 284 & 284 & 2.74 & 0 & 0 & 4 \\
oAi40A & 27 & 329 & 385 & 355 & 355 & 355 & 8.4 & 0 & 0 & 151.5 \\
oAi40B & 15 & 329 & 334 & 329 & 329 & 329 & 1.5 & 0 & 0 & 63 \\
oAi40C & 9 & 329 & 345 & 336 & 336 & 336 & 0 & 0 & 0 & 43.1 \\
oAi40D & 7 & 329 & 356 & 344 & 344 & 344 & 2.61 & 0 & 0 & 16.3 \\
\hline & & & & & & & & & & \\
\hline
\end{tabular}

\subsection{Comparison based on benchmark sets}

Table 7 reports the obtained average of all gaps and the average of computational times in seconds required for solving the problem by each method. From this table, we can conclude the following:

- For ogdb's set of instances, HACA obtained the same results as all the other methods. The gaps G-R, G-G and $\mathrm{G}-\mathrm{H}$ are equal to zero. 21 from 23 solutions (91.3\%) were proven optimal i.e. the value obtained by HACA is the same as the lower bound lb. The CPU computing time was less than one second on average.

- For oAi's set of instances, 20 from 32 solutions $(62.5 \%)$ were proven optimal (i.e. HACA = lb). The computational time average is $9.98 \mathrm{~s}$. HACA significantly performs better than RPS where G-R is $1.75 \%$. In fact, it outperforms RPS method by 16 solutions and obtains the same solutions as RPS for the remaining instances. HACA slightly performs better than both GRASP and HGA methods where $\mathrm{G}-\mathrm{G}$ and $\mathrm{G}-\mathrm{H}$ are both equal to 0.15 . It outperforms both GRASP and HGA methods by one solution and attains the same results obtained by GRASP and HGA methods for the rest of instances.

- For oBi's set of instances, 22 from 24 solutions $(91.667 \%$ ) were proven optimal (i.e. HACA = lb). The CPU computing time was $3.3 \mathrm{~s}$ on average. HACA clearly performs better than RPS where G-R is $0.44 \%$. Indeed, 
TABLE 4. Results of the HACA algorithm on oBi's instances.

\begin{tabular}{lllllllllll}
\hline \hline Instance & $M$ & lb & RPS & GRASP & HGA & HACA & G-R & G-G & G-H & $T$ \\
\hline oBi10B & 7 & 45 & 45 & 45 & 45 & 45 & 0 & 0 & 0 & $<1$ \\
oBi10C & 5 & 45 & 45 & 45 & 45 & 45 & 0 & 0 & 0 & $<1$ \\
oBi10D & 4 & 45 & 45 & 45 & 45 & 45 & 0 & 0 & 0 & $<1$ \\
oBi13B & 9 & 60 & 60 & 60 & 60 & 60 & 0 & 0 & 0 & $<1$ \\
oBi13C & 6 & 60 & 60 & 60 & 60 & 60 & 0 & 0 & 0 & $<1$ \\
oBi13D & 5 & 60 & 60 & 60 & 60 & 60 & 0 & 0 & 0 & $<1$ \\
oBi15B & 9 & 74 & 74 & 74 & 74 & 74 & 0 & 0 & 0 & 3 \\
oBi15C & 6 & 74 & 74 & 74 & 74 & 74 & 0 & 0 & 0 & $<1$ \\
oBi15D & 5 & 74 & 74 & 74 & 74 & 74 & 0 & 0 & 0 & $<1$ \\
oBi20B & 11 & 99 & 99 & 99 & 99 & 99 & 0 & 0 & 0 & $<1$ \\
oBi20C & 7 & 99 & 99 & 99 & 99 & 99 & 0 & 0 & 0 & $<1$ \\
oBi20D & 5 & 99 & 102 & 99 & 99 & 99 & 2.94 & 0 & 0 & $<1$ \\
oBi24B & 11 & 109 & 109 & 109 & 109 & 109 & 0 & 0 & 0 & 1 \\
oBi24C & 7 & 107 & 110 & 107 & 107 & 107 & 0 & 0 & 0 & $<1$ \\
oBi24D & 5 & 113 & 116 & 113 & 113 & 113 & 0 & 0 & 0 & 7 \\
oBi27B & 18 & 188 & 188 & 188 & 188 & 188 & 0 & 0 & 0 & $<1$ \\
oBi27C & 10 & 185 & 185 & 185 & 185 & 185 & 0 & 0 & 0 & 2 \\
oBi27D & 8 & 185 & 185 & 185 & 185 & 185 & 0 & 0 & 0 & 9 \\
oBi31B & 21 & 274 & 282 & 282 & 282 & 282 & 0 & 0 & 0 & 3 \\
oBi31C & 12 & 274 & 274 & 274 & 274 & 274 & 0 & 0 & 0 & $<1$ \\
oBi31D & 9 & 274 & 277 & 274 & 274 & 274 & 1.08 & 0 & 0 & 2 \\
oBi40B & 23 & 267 & 275 & 267 & 267 & 267 & 2.9 & 0 & 0 & 2 \\
oBi40C & 13 & 267 & 272 & 267 & 267 & 267 & 1.84 & 0 & 0 & 23 \\
oBi40D & 9 & 267 & 279 & 274 & 274 & 274 & 1.79 & 0 & 0 & 13 \\
\hline
\end{tabular}

it outperforms RPS method in 5 out of 24 instances and obtains the same results obtained by GRASP and HGA where $\mathrm{G}-\mathrm{G}$ and $\mathrm{G}-\mathrm{H}$ are both equal to 0 .

- For oval's set of instances, 8 from 34 solutions $(23.53 \%)$ were proven optimal (i.e. HACA $=1 b)$. The CPU computing time was $7.8 \mathrm{~s}$ on average. HACA clearly performs better than RPS where G-R is $1.64 \%$, outperforms RPS method by 28 solutions and obtains the same solutions as RPS method for the rest of instances. HACA exactly performs as both GRASP and HGA methods where the $\mathrm{G}-\mathrm{G}$ and $\mathrm{G}-\mathrm{H}$ are both equal to zero.

- For oegl's set of instances, HACA fails to reach lb for all instances (similarly as RPS, GRASP and HGA methods). The CPU computing time was $335.08 \mathrm{~s}$ on average. HACA outperforms the RPS method for all the 24 solutions and G-RPS on average is 9.45. HACA succeeds to perform better than both GRASP and HGA in one solution $(s 1 B)$. It obtains the same results as GRASP and HGA for seven solutions. The G-G is -1.81 and the $\mathrm{G}-\mathrm{H}$ is -1.94 which are considered as very slight gaps.

By analysing the results presented in Table 7, it can be deduced that our method HACA similarly performs or better than RPS, GRASP and HGA for four sets (i.e ogdb, oval, oAi and oBi), significantly outperforms RPS for oegl set and slightly gets worse results than both GRASP and HGA for some oegl's instances.

RPS, GRASP and HGA were executed at $2.4 \mathrm{GHz}$ processor while HACA was executed at $2.6 \mathrm{GHz}$ processor. To give a fair comparison that takes into consideration the different speeds of the machines used in the computational experiments, we normalized the average computational time obtained by HACA. This is done by multiplying the recorded time with the CPU speed ratio which equals to CPU speed of used machine over CPU speed of the slowest machine in other studies (i.e. $2.4 \mathrm{GHz}$ ) as proposed in $[11,13]$. The CPU speed ratio is then equal to 1.08. Hence, in Table 7, we added the column "NT-HACA" which indicates the normalized 
TABLE 5. Results of the HACA algorithm on oval's instances.

\begin{tabular}{|c|c|c|c|c|c|c|c|c|c|c|}
\hline Instance & $M$ & $\mathrm{lb}$ & RPS & GRASP & HGA & HACA & $\mathrm{G}-\mathrm{R}$ & $\mathrm{G}-\mathrm{G}$ & $\mathrm{G}-\mathrm{H}$ & $T$ \\
\hline oval1A & 4 & 146 & 154 & 149 & 149 & 149 & 3.25 & 0 & 0 & $<1$ \\
\hline oval1B & 5 & 146 & 149 & 147 & 147 & 147 & 1.43 & 0 & 0 & $<1$ \\
\hline oval1C & 10 & 146 & 146 & 146 & 146 & 146 & 0 & 0 & 0 & $<1$ \\
\hline oval2A & 4 & 185 & 195 & 189 & 189 & 189 & 3.08 & 0 & 0 & $<1$ \\
\hline oval2B & 5 & 185 & 192 & 186 & 186 & 186 & 3.13 & 0 & 0 & $<1$ \\
\hline oval2C & 10 & 185 & 185 & 185 & 185 & 185 & 0 & 0 & 0 & $<1$ \\
\hline oval3A & 4 & 65 & 71 & 67 & 67 & 67 & 5.63 & 0 & 0 & $<1$ \\
\hline oval3B & 5 & 65 & 67 & 66 & 66 & 66 & 0 & 0 & 0 & $<1$ \\
\hline oval3C & 9 & 65 & 65 & 65 & 65 & 65 & 1.49 & 0 & 0 & $<1$ \\
\hline oval4A & 5 & 344 & 358 & 350 & 350 & 350 & 2.23 & 0 & 0 & $<1$ \\
\hline oval4B & 6 & 343 & 354 & 347 & 347 & 347 & 1.98 & 0 & 0 & 6 \\
\hline oval4C & 7 & 343 & 350 & 345 & 345 & 345 & 1.43 & 0 & 0 & $<1$ \\
\hline oval4D & 11 & 343 & 346 & 343 & 343 & 343 & 0.87 & 0 & 0 & $<1$ \\
\hline oval5A & 5 & 367 & 381 & 374 & 374 & 374 & 0 & 0 & 0 & $<1$ \\
\hline oval5B & 6 & 367 & 376 & 371 & 371 & 371 & 1.84 & 0 & 0 & 2 \\
\hline oval5C & 7 & 367 & 372 & 368 & 368 & 368 & 1.08 & 0 & 0 & 3 \\
\hline oval5D & 11 & 367 & 370 & 367 & 367 & 367 & 0.82 & 0 & 0 & $<1$ \\
\hline oval6A & 5 & 190 & 195 & 192 & 192 & 192 & 1.54 & 0 & 0 & 2 \\
\hline oval6B & 6 & 190 & 192 & 191 & 191 & 191 & 0.52 & 0 & 0 & $<1$ \\
\hline oval6C & 12 & 190 & 190 & 190 & 190 & 190 & 0 & 0 & 0 & $<1$ \\
\hline oval7A & 5 & 249 & 263 & 256 & 256 & 256 & 2.66 & 0 & 0 & 1 \\
\hline oval7B & 6 & 249 & 259 & 253 & 253 & 253 & 2.32 & 0 & 0 & 7 \\
\hline oval7C & 11 & 249 & 250 & 249 & 249 & 249 & 0.4 & 0 & 0 & 32 \\
\hline oval8A & 5 & 347 & 359 & 354 & 354 & 354 & 1.39 & 0 & 0 & 2 \\
\hline oval8B & 6 & 347 & 354 & 351 & 351 & 351 & 0.85 & 0 & 0 & 4 \\
\hline oval8C & 11 & 347 & 348 & 347 & 347 & 347 & 0.29 & 0 & 0 & 8 \\
\hline oval9A & 5 & 278 & 299 & 292 & 292 & 292 & 2.34 & 0 & 0 & 19 \\
\hline oval9B & 6 & 278 & 298 & 290 & 290 & 290 & 2.68 & 0 & 0 & 7 \\
\hline oval9C & 7 & 278 & 294 & 288 & 288 & 288 & 2.04 & 0 & 0 & 13 \\
\hline oval9D & 12 & 278 & 288 & 280 & 280 & 280 & 0 & 0 & 0 & 21 \\
\hline oval10A & 5 & 376 & 403 & 391 & 391 & 391 & 2.98 & 0 & 0 & 9 \\
\hline oval10B & 6 & 376 & 399 & 388 & 388 & 388 & 2.76 & 0 & 0 & 34 \\
\hline oval10C & 7 & 376 & 394 & 385 & 385 & 385 & 2.28 & 0 & 0 & 23 \\
\hline oval10D & 12 & 376 & 387 & 377 & 377 & 377 & 2.58 & 0 & 0 & 57 \\
\hline
\end{tabular}

average running time of HACA. It is clear that HACA is many times faster than RPS, GRASP and HGA for all instances. For example, the overall time performance for oegl's set of instances is 5.49, 2.86 and 2.67 faster than RPS, GRASP and HGA respectively.

Table 8 shows for each set of instances and for each method the number of same solutions with the same cost, the number of worse solutions and the number of better solutions in comparison with HACA. Table 8 shows for each set of instances and for each method the number of same solutions with the same cost, the number of worse solutions and the number of better solutions in comparison with HACA. HACA overcomes RPS in 74 instances and obtains the same solutions in 63 instances. HACA outperforms GRASP in one instance, obtains the same solutions in 120 instances and is overcame by GRASP in 16 solutions. Finally, HACA outperforms HGA in one instance, obtains the same solutions as it in 120 instances and is outperformed by HGA in 16 solutions. 
TABLE 6. Results of the HACA algorithm on oegl's instances.

\begin{tabular}{lllllllllll}
\hline \hline Instance & $M$ & lb & RPS & GRASP & HGA & HACA & G-R & G-G & G-H & $T$ \\
\hline oegl-e1-A & 7 & 1590 & 1755 & 1659 & 1659 & 1659 & 5.47 & 0 & 0 & 99 \\
oegl-e1-B & 9 & 1524 & 1726 & 1589 & 1589 & 1589 & 7.94 & 0 & 0 & 125 \\
oegl-e1-C & 12 & 1490 & 1610 & 1542 & 1542 & 1542 & 4.22 & 0 & 0 & 165 \\
oegl-e2-A & 9 & 1965 & 2256 & 2035 & 2035 & 2043 & 9.44 & -0.39 & -0.39 & 119 \\
oegl-e2-B & 12 & 1912 & 2166 & 1971 & 1971 & 1971 & 9 & 0 & 0 & 181 \\
oegl-e2-C & 16 & 1879 & 2151 & 1964 & 1964 & 1964 & 8.69 & 0 & 0 & 189 \\
oegl-e3-A & 10 & 2245 & 2676 & 2372 & 2366 & 2382 & 10.99 & -0.42 & -0.68 & 171 \\
oegl-e3-B & 14 & 2203 & 2596 & 2321 & 2321 & 2350 & 9.48 & -1.25 & -1.25 & 286 \\
oegl-e3-C & 19 & 2188 & 2565 & 2270 & 2260 & 2280 & 11.11 & -0.44 & -0.88 & 181 \\
oegl-e4-A & 11 & 2453 & 2825 & 2556 & 2554 & 2573 & 8.92 & -0.67 & -0.74 & 344 \\
oegl-e4-B & 16 & 2453 & 2853 & 2517 & 2517 & 2537 & 11.08 & -0.79 & 0.79 & 237 \\
oegl-e4-C & 21 & 2453 & 2805 & 2491 & 2497 & 2524 & 10.02 & -1.32 & -1.08 & 371 \\
oegl-s1-A & 9 & 1503 & 1787 & 1604 & 1604 & 1604 & 10.24 & 0 & 0 & 399 \\
oegl-s1-B & 12 & 1426 & 1729 & 1579 & 1579 & 1565 & 9.49 & 0.89 & 0.89 & 144 \\
oegl-s1-C & 16 & 1397 & 1757 & 1512 & 1512 & 1512 & 13.94 & 0 & 0 & 222 \\
oegl-s2-A & 16 & 3205 & 4068 & 3567 & 3566 & 3665 & 9.91 & -2.75 & -2.78 & 354 \\
oegl-s2-B & 22 & 3174 & 4009 & 3442 & 3428 & 3595 & 10.33 & -4.45 & -4.87 & 392 \\
oegl-s2-C & 29 & 3174 & 3904 & 3341 & 3340 & 3534 & 9.48 & -5.78 & -5.81 & 651 \\
oegl-s3-A & 17 & 3381 & 4242 & 3734 & 3704 & 3776 & 10.99 & -1.12 & -1.94 & 612 \\
oegl-s3-B & 24 & 3379 & 4158 & 3564 & 3558 & 3704 & 10.92 & -3.93 & -4.1 & 439 \\
oegl-s3-C & 31 & 3379 & 4102 & 3492 & 3495 & 3588 & 12.53 & -2.75 & -2.66 & 197 \\
oegl-s4-A & 21 & 4186 & 4965 & 4409 & 4399 & 4544 & 8.48 & -3.06 & -3.3 & 484 \\
oegl-s4-B & 29 & 4186 & 4973 & 4323 & 4312 & 4523 & 9.05 & -4.63 & -4.89 & 552 \\
oegl-s4-C & 37 & 4186 & 5019 & 4309 & 4282 & 4536 & 9.62 & -5.27 & -5.93 & 1128 \\
\hline
\end{tabular}

TABLE 7. Gap and time comparisons of HACA with RPS, GRASP and HGA.

\begin{tabular}{lllllllll}
\hline \hline Set & G-R & G-G & G-H & T-RPS & T-GRASP & T-HGA & T-HACA & NT-HACA \\
\hline ogdb & 0 & 0 & 0 & 66.2 & $<0.2$ & $<0.1$ & $<0.1$ & $<1$ \\
oAi & 1.63 & 0 & 0 & 447.7 & 17.2 & 40.1 & 9.98 & 10.077 \\
oBi & 0.44 & 0 & 0 & 345.8 & 32 & 32 & 3.3 & 3.564 \\
oval & 1.64 & 0 & 0 & 756.1 & 71.8 & 12 & 7.8 & 8.42 \\
oegl & 9.45 & -1.81 & -1.94 & 1989.6 & 1034.5 & 968.1 & 335.08 & 361.9 \\
\hline
\end{tabular}

TABLE 8. Comparison of different obtained solutions of HACA with RPS, GRASP and HGA.

\begin{tabular}{lllllllllll}
\hline \hline & & \multicolumn{3}{c}{ RPS } & \multicolumn{3}{c}{ GRASP } & \multicolumn{3}{c}{ HGA } \\
\cline { 3 - 10 } Set & nb & Same & Worse & Better & Same & Worse & Better & Same & Worse & Better \\
\hline ogld & 23 & 23 & 0 & 0 & 23 & 0 & 0 & 23 & 0 & 0 \\
oAi & 32 & 16 & 0 & 16 & 32 & 0 & 0 & 32 & 0 & 0 \\
oBi & 24 & 18 & 0 & 6 & 24 & 0 & 0 & 24 & 0 & 0 \\
oval & 34 & 6 & 0 & 28 & 34 & 0 & 0 & 34 & 0 & 0 \\
oegl & 24 & 0 & 0 & 24 & 7 & 16 & 1 & 7 & 16 & 1 \\
\hline Total & 137 & 63 & 0 & 74 & 120 & 16 & 1 & 120 & 16 & 1 \\
\hline
\end{tabular}


TABLE 9. Comparison of HACA with RPS, GRASP and HGA.

\begin{tabular}{llll}
\hline \hline & Class 1 & Class 2 & Class 3 \\
\cline { 2 - 4 } & $\left|E_{r}\right|<50$ & $50 \leq\left|E_{r}\right| \leq 100$ & $\left|E_{r}\right|>100$ \\
\hline ogdb & 22 & 1 & 0 \\
oAi & 24 & 8 & 0 \\
oBi & 21 & 3 & 0 \\
oval & 9 & 25 & 0 \\
oegl & 0 & 15 & 9 \\
\hline Total & 76 & 52 & 9 \\
\hline G-R & 0.45 & 3.9 & 9.63 \\
G-G & 0 & -0.08 & -4.35 \\
G-H & 0 & -0.09 & -4.63 \\
\hline Final gap & 0.15 & 1.24 & 0.21 \\
\hline
\end{tabular}

\subsection{Comparison based on instance sizes}

The purpose of this subsection is to provide a new comparison of experiment results based on the size of the instances. Indeed, there is still a need to make a reasonably consistent and fair comparison taking into consideration the number of tasks in each instance. To do so, we classify all instances presented in Section 4.1 into three classes according to the number of tasks $\left|E_{r}\right|$. The first class contains all instances whose number of tasks is less than 50. The second class contains all instances whose number of tasks is between 50 and 100 . The third class contains all instances whose number of tasks is greater than 100. Table 9 represents the number of instances in each class and the new gaps. The total number of instances belonging to the first class is 76 and HACA reaches a much better performance than RPS, GRASP and HGA where the gaps are respectively 0.45 , 0 and 0 . The total number of instances belonging to the second class is 52 and HACA gets better results than RPS ( $\mathrm{G}-\mathrm{R}$ is 3.9) and obtains almost the same results as GRASP and HGA where both gaps $\mathrm{G}-\mathrm{G}(-0.08)$ and G-H (-0.09) are of negligible significance. Finally, the number of instances in the third class is 9 and HACA succeeds to surpass RPS where G-R is 9.63 and slightly gets worse than GRASP and HGA with G-G is -4.35 and $\mathrm{G}-\mathrm{H}$ is -4.63 .

The experiments that we conducted using HACA have shown the production of a population of infeasible solutions at high rates because of number of vehicles constraint. The algorithm maintains the population in only the feasible search space, thus excluding infeasible solutions during the algorithm evolution. This significantly reduces the quality of solutions since the search space is restricted to feasible region. Thus, our proposed algorithm fails to find solutions for some instances with $M^{*}$ and $M^{*}+1$ and when finding such solutions, their costs are not competitive with those reported in [1]. The authors in [1] showed that the feasibility rate for almost all instances with the number of vehicles $M^{*}$ and $M^{*}+1$ is very small and this rate is higher when running large-scale problems (i.e. Eglese's instances). Then, they equipped their method with a feasibility technique in order to overcome the presence of infeasible solutions with respect to the number of vehicles. That justifies our choice to perform our experiments only with number of vehicles $M^{*}+2$ and why our algorithm HACA works efficiently and succeeds to obtain competitive results with both GRASP and HGA methods for small and medium instances (as shown in both class 1 and class 2 - Tab. 9), but fails to obtain good quality solutions for large instances (as shown in class 3 - Tab. 9).

\subsection{Parameter setting}

For each set of instances, several runs of the program with different parameter values were performed and the best obtained values are summarized in the following. The values of $N_{a}, \alpha, \beta, \rho$ and $q_{0}$ are found and recommended by Dorigo et al. [5]. We use between 500 and 2500 maximum iterations $N_{\text {iter }}$ in HACA algorithm. 
In the Simulated Annealing algorithm, $K$ is tested between 0.5 and $0.9, T_{\max }$ is tested between 180 and 260 and $T_{\min }$ is tested between 10 and 30 . The parameter values obtaining the best improvement solutions with an acceptable computational time are $N_{a}=20, \alpha=1, \beta=2, \rho=0.85, q_{0}=0.6, N_{\text {iter }}=1750, K=0.7, T_{\max }=200$ and $T_{\min }=10$.

\section{Conclusion}

Open Capacitated Arc Routing Problem (OCARP) has important real-world applications like the Meter Reader Routing Problem (MRRP), but it has not been extensively studied in literature. We have adapted a constructive heuristic to find an initial solution for OCARP. We used the strategy of a giant route and then an optimal splitting method is applied to make the solution feasible and then evaluate it. We have proposed an ant colony algorithm hybridized with a local search method based on four important moves to solve this problem. We have presented results for different OCARP instances on five different sets from literature. The obtained results are very good in comparison with the well-known Reactive Path Scanning (RPS) which is guided by a cost-demand edge-selection and ellipse rules. Comparing the solution quality of the proposed algorithm with the existing meta-heuristics GRASP and HGA in literature, the computational results indicate that HACA performs very good and provides competitive results.

Extending description of OCARP problem to cover more operational constraints such as maximum worker time or customer time windows could be a promising direction to be more studied in the future. We also intend to equip our approach with a feasibility method in order to tackle instances with number of vehicles $M^{*}$ and $M^{*}+1$.

Acknowledgements. This work has been jointly funded with the support of the National Council for Scientific Research in Lebanon CNRS-L and Lebanese University.

\section{REFERENCES}

[1] R.K. Arakaki and F.L. Usberti, Hybrid genetic algorithm for the open capacitated arc routing problem. Comput. Oper. Res. 90 (2018) 221-231.

[2] E. Benavent, V. Campos, A. Corberán and E. Mota, The capacitated arc routing problem: lower bounds. Networks 22 (1992) 669-690.

[3] A. Colorni, M. Dorigo and V. Maniezzo, Distributed optimization by ant colonies (1991) 134-142.

[4] T.H. Cormen, C.E. Leiserson, R.L. Rivest and C. Stein, Introduction to Algorithms, 3rd edition. The MIT Press (2009).

[5] M. Dorigo, V. Maniezzo and A. Colorni, Ant system: optimization by a colony of cooperating agents. IEEE Trans. Syst. Man Cybern. Part B (Cybern.) $\mathbf{2 6}$ (1996) 29-41.

[6] R.W. Eglese, Routing winter gritting vehicles. Disc. Appl. Math. 48 (1994) 231-244.

[7] B.L. Golden and R.T. Wong, Capacitated arc routing problems. Networks 11 (1981) 305-315.

[8] B.L. Golden, J.S. Dearmon and E.K. Baker, Computational experiments with algorithms for a class of routing problems. Comput. Oper. Res. 10 (1983) 47-59.

[9] S. Kirkpatrick, C.D. Gelatt and M.P. Vecchi, Optimization by simulated annealing. Science 220 (1983) 671-680.

[10] P. Lacomme, C. Prins and W. Ramdane-Chérif, Evolutionary algorithms for periodic arc routing problems. Eur. J. Oper. Res. 165 (2005) 535-553. Project Management and Scheduling.

[11] L. Muyldermans and G. Pang, A guided local search procedure for the multi-compartment capacitated arc routing problem. Comput. Oper. Res. 37 (2010) 1662-1673.

[12] I.H. Osman and C.N. Potts, Simulated annealing for permutation flow-shop scheduling. Omega 17 (1989) $551-557$.

[13] L. Santos, J. Coutinho-Rodrigues and J.R. Current, An improved ant colony optimization based algorithm for the capacitated arc routing problem. Trans. Res. B: Methodol. 44 (2010) 246-266.

[14] H.I. Stern and M. Dror, Routing electric meter readers. Comput. Oper. Res. 6 (1979) 209-223.

[15] T. Stutzle and M. Dorigo, A short convergence proof for a class of ant colony optimization algorithms. IEEE Trans. Evol. Comput. 6 (2002) 358-365.

[16] G. Ulusoy, The fleet size and mix problem for capacitated arc routing. Eur. J. Oper. Res. 22 (1985) $329-337$.

[17] F.L. Usberti, P.M. França and A.L.M. França, The open capacitated arc routing problem. Comput. Oper. Res. 38 (2011) 1543-1555.

[18] F.L. Usberti, P.M. França and A.L.M. França, Branch-and-bound algorithm for an arc routing problem. Annals XLIV SBPO, Rio de Janeiro (2012). 
[19] F.L. Usberti, P.M. França and A.L.M. França, Grasp with evolutionary path-relinking for the capacitated arc routing problem. Comput. Oper. Res. 40 (2013) 3206-3217.

[20] P.J.M. van Laarhoven, E.H.L. Aarts and J.K. Lenstra, Job shop scheduling by simulated annealing. Oper. Res. 40 (1992) 113-125.

[21] J. Wunderlich, M. Collette, L. Levy and L. Bodin, Scheduling meter readers for Southern California gas company. INFORMS J. Appl. Anal. 22 (1992) 22-30. 